\title{
Transvaginal Real-Time Shear Wave Elastography in the Diagnosis of Endometrial Lesions
}

\author{
Yuan-Yuan Du \\ Xiao-jing Yan \\ Yan-Jing Guo \\ Jing Wang \\ Xiao-Duo Wen \\ Nan Wang \\ Yi Yang
}

Department of Gynecology and Obstetrics Ultrasound, The Fourth Hospital of Hebei Medical University, Shijiazhuang, 0500II, People's Republic of China

Correspondence: Yi Yang

Department of Gynecology and Obstetrics Ultrasound, The Fourth Hospital of Hebei

Medical University, No. 12 of Jiankang Road, Chang'an District, Shijiazhuang, 0500I I,

People's Republic of China

$\mathrm{Tel} / \mathrm{Fax}+8631 \mathrm{I} 86095378$

Email yangyi_dr336@163.com
Background: To explore the value of transvaginal real-time shear wave elastography (SWE) in the diagnosis of endometrial lesions.

Methods: A total of 140 female patients with endometrial lesions, confirmed by pathological results, were divided into three groups: 45 cases of endometrial polyps, 29 cases of endometrial hyperplasia and 66 cases of endometrial cancer. A total of 100 cases of normal endometrium were used as the control group, including 52 cases in the proliferative stage and 48 cases in the secretory stage. Transvaginal real-time shear wave elastography was performed in all four groups.

Results: Emean, Emax and Esd were expressed as the average standard deviation. Among the control group, the results were $26.24 \pm 9.74,38.09 \pm 9.18$, and $4.25 \pm 2.73 \mathrm{kPa}$, respectively, in the proliferative endometrium cases and $12.51 \pm 7.46,27.22 \pm 11.32,4.40 \pm 2.52 \mathrm{kPa}$, respectively, in the secretory endometrium cases. Among the experimental group, the result was $15.68 \pm 8.18,27.28 \pm 10.28$ and $3.62 \pm 1.81 \mathrm{kPa}$ respectively in the endometrial polyps cases; $21.20 \pm 12.57,36.32 \pm 15.04$, and $5.09 \pm 3.93 \mathrm{kPa}$ in the endometrial hyperplasia cases; 49.36 $\pm 25.51,86.66 \pm 42.27$ and $14.86 \pm 10.63 \mathrm{kPa}$ in the endometrial cancer cases. The difference was statistically significant $(\mathrm{P}<0.05)$. When the truncation values of Emean, Emax and Esd were $28.50,52.45$ and $9.05 \mathrm{kPa}$, respectively, to distinguish between normal endometrium and endometrial cancer, Emax has the highest diagnostic value.

Conclusion: Real-time SWE technology might be used as an auxiliary method in the diagnosis and differential diagnosis of endometrial cancer. More quantitative indicators are conducive to diagnosis.

Keywords: ultrasound, endometrial lesions, real-time shear wave elastography, differential diagnosis

\section{Background}

Benign endometrial lesions mainly include endometrial polyps and endometrial hyperplasia, and malignant endometrial lesions mainly include endometrial cancer and endometrial sarcoma. There are some similar clinical symptoms in benign and malignant lesions: irregular vaginal bleeding, abnormal vaginal discharge, and abdominal pain. ${ }^{1}$ Accumulating evidence has reported that cell cycle quiescence of stem cells which lead by inflammatory cytokines and reactive oxygen species may be related with the development of benign and malignant endometrial aberrations. $^{2-4}$ The clinical methods for differentiating benign and malignant endometrial lesions mainly include ultrasound, computed tomography (CT), magnetic resonance imaging (MRI), endometrial biopsy, and hysteroscopy. Among these methods, CT has the disadvantage of radiation exposure, whilst, although MRI 
does not use radiation and is considered as a non-invasive procedure, the examination time is longer and the cost is comparatively higher. Therefore, CT and MRI are not routine gynecological examination methods. Diagnostic curettage and hysteroscopy have advantages in obtaining specimens for pathological diagnosis and have a lower false-positive rate than other imaging methods, however, as invasive interventions, they may cause complications such as intrauterine adhesions, bleeding and infection. Moreover, because of the fluidity of the physiological saline medium in hysteroscopy, cancer cells may be implanted into the pelvis or even abdominal cavity through the bilateral fallopian tubes. Therefore, diagnostic curettage and hysteroscopy are not the preferred methods for disease screening. ${ }^{5}$ As a non-invasive approach, ultrasound is valued for its safe, rapid, reproducible, and inexpensive qualities. Compared with traditional transabdominal ultrasound, transvaginal ultrasound does not require the bladder to be filled and is less affected by abdominal fat and intestinal gas. Meanwhile, the intracavity probe has a high frequency and resolution and can display pelvic lesions more clearly. These advantages have made transvaginal ultrasound the preferred method of gynecological ultrasound. But for some atypical endometrial lesions, ultrasound has a higher false-positive rate, ${ }^{6-10}$ which is a challenge for doctors who lack experience. Therefore, it is particularly urgent to find new ultrasound technology for more convenient diagnosis and differential diagnosis of endometrial lesions.

Real-time shear wave elastography (SWE) is a new ultrasonic technology. It works by continuously focusing the tissue at different depths by transmitting the acoustic radiation pulse of the safety range through the probe. The transverse shear wave is generated by high-frequency vibration. The shear wave is detected by ultra-high-speed imaging technology, and Young's modulus ${ }^{11}$ is obtained by the quantitative analysis system. At the same time, the elastic hardness map of the tissue is displayed in real-time using color-coding. Young's modulus is calculated in terms of the density of tissue, shear wave velocity (SWV) and $\mathrm{kPa}$. Young's modulus value can reflect the hardness of the tissue: the larger the value of Young's modulus is, the faster the SWV is and the greater the elastic hardness of the tissue becomes. ${ }^{12}$ This new technology has real-time, objective and repeatable characteristics. It has been widely used in diagnosing thyroid, breast, liver, kidney, lymph node, and carotid artery diseases. ${ }^{12-16}$ In gynecology, there have been research studies performed on the uterine wall and cervix, ${ }^{17-19}$ however, studies on normal endometrium and endometrial lesions are relatively rare. ${ }^{20}$

The purpose of this study was to explore the value of real-time transvaginal SWE in the diagnosis and differential diagnosis of endometrial lesions, and to obtain quantitative indicators for the diagnosis of endometrial cancer.

\section{Methods}

\section{Study Population}

A total of 140 patients with suspected endometrial lesions were treated in the Fourth Hospital of Hebei Medical University from May 2018 to February 2019. All these patients underwent transvaginal ultrasound and a real-time SWE examination before the operation. The results of their pathological examinations were obtained during the hospital operation and subsequent follow-up. A total of 100 patients with normal physical examination were selected as the control group, all of whom were women of childbearing age with normal menstruation and underwent transvaginal ultrasound and real-time SWE examination. The control group was divided into proliferative endometrium group and secretory endometrium group. The inclusion criteria included: (1) endometrial lesions must have been confirmed by surgery or biopsy pathological results; (2) endometrial thickness $\geq$ $5 \mathrm{~mm}$. Exclusion criteria included: (1) patients receiving chemotherapy or radiotherapy; (2) patients who had a curettage; (3) patients with an IUD; (4) patients receiving estrogen replacement therapy. This study was conducted with approval from the Ethics Committee of the Fourth Hospital of Hebei Medical University, and conducted in accordance with the declaration of Helsinki. Written informed consent was obtained from all participants.

\section{Study Procedures}

A SuperSonic Imagine AixPlorer ultrasound system was used with an intracavitary probe frequency of $3 \sim 12 \mathrm{MHz}$.

Before the examination, empty urinary bladder was required. Patients should take the lithotomy position. Then, a protective condom was put on the intracavitary probe. The transvaginal two-dimensional ultrasonography image was made by placing the probe in the vaginal fornix. Thus, the thickness, shape, texture, blood flow and endometrial lesions of the endometrium could be observed and recorded according to the menstrual cycle of the patients. Generally, the longitudinal section of the uterus was switched to SWE mode, and the region of interest (ROI) was covered with 
normal intima (or lesion) as far as possible, avoiding the fluid area, calcification area, blood vessel and other structures. Patients were required to breathe with a light screen for 3 5 seconds in a quiet breathing state. Images were frozen after stabilization, and through playing back images, the best images were taken in color signal to measure. An adjusted round Q-box was used when measuring. In other words, the circular Q-box was adjusted to the maximum diameter in the normal intima (or lesion), and the normal intima (or lesion) was encapsulated as much as possible. The mean Young's modulus (Emean), the maximum Young's modulus (Emax) and the standard deviation of Young's modulus (Esd) were obtained. The values were measured three times and averaged.

\section{Statistical Analysis}

SPSS19.0 statistical analysis software was used for data analysis. The measurement data were represented by average standard deviation $(\bar{x} \pm s)$. The comparison between the groups was performed by $t$-test or by variance analysis. The Kruskal-Wallis test in the nonparametric test was applied to the measurement data with uneven variance. When $P<0.05$, there was a statistically significance. A receiver operating characteristic (ROC) curve was drawn to evaluate the diagnostic value.

\section{Results}

The general information of the experimental group and the control group are comparable $(P>0.05)$. Of these 140 patients with suspected endometrial lesions, there were 45 cases of endometrial polyps, where the average age of the patient was $45.71 \pm 9.68$ years old; 29 cases of endometrial hyperplasia, where the average age of the patient was 44.03 \pm 8.54 years old; 66 cases of endometrial carcinoma, where the average age of the patient was $55.49 \pm 11.41$ years old. There were also 58 cases of endometrial adenocarcinoma, 5 cases of atypical endometrial hyperplasia, 1 case of cervical non-keratinizing squamous cell carcinoma, 1 case of endometrial clear cell carcinoma, and 1 case of uterine carcinoma. There were 100 cases of normal endometrium, among which 52 cases were in the proliferative stage, where the average age of the patient was 33.89 \pm 7.81 years old, and 48 cases were in the secretory stage, where the average age of the patient was $34.48 \pm 9.64$ years old.

Real-time SWE manifestations of the proliferative endometrium were as follows: the functional layer was comparatively uniform blue, and the basal layer and uterine line were comparatively lighter (Figure 1A). Emean, Emax and Esd of the endometrium in the proliferative stage were $26.24 \pm 9.74$ $\mathrm{kPa}, 38.09 \pm 9.18 \mathrm{kPa}$, and $4.25 \pm 2.73 \mathrm{kPa}$, respectively.

Real-time SWE manifestations of the endometrium in the secretory phase were as follows: the demarcation color between the functional layer and the basal layer was not obvious, which was uniform blue, and the color of the uterine cavity line was slightly lighter (Figure 1B). Emean, Emax and Esd of the endometrium in the secretory stage were 12.51 $\pm 7.46 \mathrm{kPa}, 27.22 \pm 11.32 \mathrm{kPa}$, and $4.40 \pm 2.52 \mathrm{kPa}$ respectively.

Real-time SWE manifestations of the endometrial polyps were as follows: the lesions were uniform blue (Figure 1C), Emean, Emax and Esd were 15.68 \pm 8.18 $\mathrm{kPa}, 27.28 \pm 10.28 \mathrm{kPa}$, and $3.62 \pm 1.81 \mathrm{kPa}$, respectively.

Real-time SWE manifestations of endometrial hyperplasia were as follows: the lesion endometrium was relatively uniform blue (Figure 1D). Emean, Emax and Esd were $21.20 \pm 12.57 \mathrm{kPa}, 36.32 \pm 15.04 \mathrm{kPa}$, and $5.09 \pm$ $3.93 \mathrm{kPa}$, respectively.

Real-time SWE manifestations of endometrial cancer were as follows: the demarcation color between the lesion and surrounding tissues is not clear, which is yellow-blue mixed, even red-yellow-blue mixed. When the lesion involved the cervix, the main color of the affected cervix changed from blue to yellow, or even yellow-red mixed (Figure 1E). Emean, Emax and Esd were 49.36 \pm 25.51 $\mathrm{kPa}, 86.66 \pm 42.27 \mathrm{kPa}$, and $14.86 \pm 10.63 \mathrm{kPa}$, respectively.

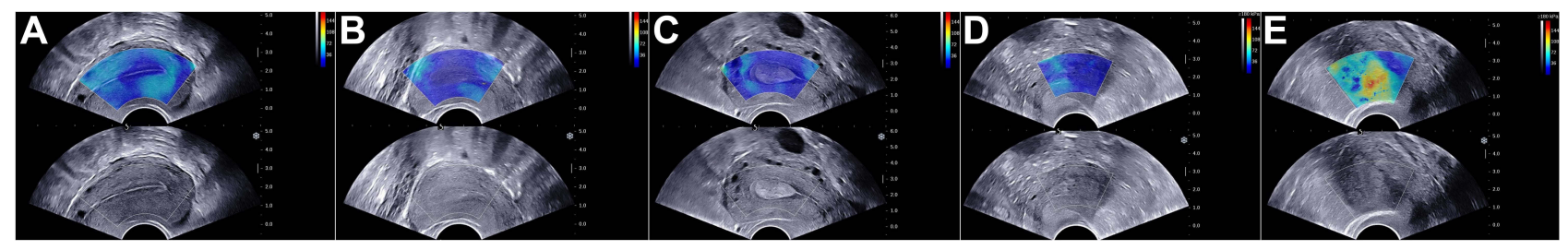

Figure I (A) Ultrasound image of proliferative endometrium in the figure. SWE showed a relatively uniform blue area in the endometrium. (B) Ultrasound image of secretory endometrium. SWE showed a uniform blue area in the endometrium. (C) Pathologic results confirmed an endometrial polyp in the uterine cavity. SWE showed the poly was relatively uniform blue. (D) Pathologic results confirmed hyperplastic endometrium. SWE showed the endometrium was blue. (E) Pathologic results confirmed an endometrial cancer in the uterine cavity. SWE showed tumor lesions of a mixture of red, yellow and blue. 
Table I Comparison of Emean, Emax and Esd Between Endometrial Carcinoma and Normal Endometrium (Average \pm Standard Deviation)

\begin{tabular}{|l|l|l|l|l|}
\hline Groups (Cases) & Emean $\mathbf{( k P a )}$ & Emax $\mathbf{( k P a )}$ & Esd (kPa) & Age \\
\hline Normal endometrium (100) & $19.65 \pm I I .08$ & $32.87 \pm I I .58$ & $4.32 \pm 2.62$ & $34.17 \pm 8.70$ \\
Endometrial carcinoma (66) & $49.36 \pm 25.5 \mathrm{I}$ & $86.66 \pm 42.27$ & $14.86 \pm 10.63$ & $55.49 \pm 11.41$ \\
$P$ & $<0.05$ & $<0.05$ & $<0.05$ & $<0.05$ \\
\hline
\end{tabular}

Table 2 Comparison of Emean, Emax and Esd Among Groups of Endometrial Carcinoma, Proliferative Endometrium, and Secretory Endometrium (Average \pm Standard Deviation)

\begin{tabular}{|l|l|l|l|l|}
\hline Groups (Cases) & Emean (kPa) & Emax (kPa) & Esd (kPa) & Age \\
\hline Proliferative endometrium (52) & $26.24 \pm 9.74 *$ & $38.09 \pm 9.18^{*}$ & $4.25 \pm 2.73 \%$ & $33.89 \pm 7.8 I^{*}$ \\
Secretory endometrium (48) & $12.51 \pm 7.46^{\#}$ & $27.22 \pm 11.32^{\#}$ & $4.40 \pm 2.52^{*}$ & $34.48 \pm 9.64^{\star}$ \\
Endometrial carcinoma (66) & $49.36 \pm 25.5 I^{*}$ & $86.66 \pm 42.27^{*}$ & $14.86 \pm 10.63^{*}$ & $55.49 \pm I I .4 I^{*}$ \\
\hline
\end{tabular}

Notes: For each column, compared between $* *$ and ${ }^{\#}, P<0.05$; for each column, compared between ${ }^{\#}$ and ${ }^{\#}$ or ${ }^{*}$ and ${ }^{*}, P>0.05$.

Table 3 Comparison of Emean, Emax and Esd Among Groups of Endometrial Carcinoma, Endometrial Polyp, and Endometrial Hyperplasia (Average \pm Standard Deviation)

\begin{tabular}{|l|l|l|l|l|}
\hline Groups (Cases) & Emean $(\mathbf{k P a})$ & Emax (kPa) & Esd (kPa) & Age \\
\hline Endometrial polyp (45) & $15.68 \pm 8.18^{\#}$ & $27.28 \pm 10.28^{\#}$ & $3.62 \pm 1.81^{\#}$ & $45.71 \pm 9.68^{\#}$ \\
Endometrial hyperplasia (29) & $21.20 \pm 12.57^{\#}$ & $36.32 \pm 15.04^{\#}$ & $5.09 \pm 3.93^{\#}$ & $44.03 \pm 8.54^{\#}$ \\
Endometrial carcinoma (66) & $49.36 \pm 25.51^{*}$ & $86.66 \pm 42.27^{*}$ & $14.86 \pm 10.63^{*}$ & $55.49 \pm 11.41^{*}$ \\
\hline
\end{tabular}

Notes: For each column, compared between * and ${ }^{\#}, P<0.05$; for each column, compared between ${ }^{\#}$ and ${ }^{\#}, P>0.05$.

Table 4 Comparison of Emean, Emax and Esd Among Groups of Proliferative Endometrium, Secretory Endometrium, Endometrial Polyp, and Endometrial Hyperplasia (Average \pm Standard Deviation)

\begin{tabular}{|l|l|l|l|l|}
\hline Groups (Cases) & Emean (kPa) & Emax (kPa) & Esd (kPa) & Age \\
\hline Proliferative endometrium (52) & $26.24 \pm 9.74^{*}$ & $38.09 \pm 9.18^{*}$ & $4.25 \pm 2.73^{\#}$ & $33.89 \pm 7.81^{*}$ \\
Secretory endometrium (48) & $12.51 \pm 7.46^{\#}$ & $27.22 \pm 11.32^{\#}$ & $4.40 \pm 2.52^{\#}$ & $34.48 \pm 9.64^{*}$ \\
Endometrial polyp (45) & $15.68 \pm 8.18^{\#}$ & $27.28 \pm 10.28^{\#}$ & $3.62 \pm 1.81^{\#}$ & $45.71 \pm 9.68^{\#}$ \\
Endometrial hyperplasia (29) & $21.20 \pm 12.57^{\#}$ & $36.32 \pm 15.04^{\#}$ & $5.09 \pm 3.93^{\#}$ & $44.03 \pm 8.54^{\#}$ \\
\hline
\end{tabular}

Notes: For each column, compared between ${ }^{*}$ and ${ }^{\#}, P<0.05$; for each column, compared between ${ }^{*}$ and ${ }^{\#}, P>0.05$.

The normal endometrium group, polyp group, hyperplasia group and cancer group were compared, with Emean, Emax and Esd as observation indicators. The difference was statistically significant $(\mathrm{P}<0.05)$. For further comparison, the results are shown in Tables 1-4.

Further analysis of endometrial cancer and normal endometrium: The area under the ROC curve (AUC) of Emean, Emax and Esd was 0.87, 0.96 and 0.90, respectively (Figure 2), which was used to distinguish normal endometrium from endometrial cancer $(\mathrm{P}<0.05)$. Sensitivity, specificity, positive predictive value, negative predictive value and accuracy were $80.3 \%, 76.0 \%, 68.8 \%$,
$85.4 \%$, and $77.7 \%$ respectively when Emean was 28.50 $\mathrm{kPa}$ as the cut-off value, $83.3 \%, 98.0 \%, 96.5 \%, 89.9 \%$, $92.2 \%$, respectively when Emax was $52.45 \mathrm{kPa}$ as the cutoff value, and $69.7 \%, 95.0 \%, 90.2 \%, 82.6 \%$ and $84.9 \%$ respectively when Esd was $9.05 \mathrm{kPa}$ as the cut-off value (Table 5).

\section{Discussion}

Increasing methods have been applied in the diagnosis of Endometrial lesions, including real-time SWE, 3D transvaginal ultrasound, and color Doppler., ${ }^{9,22}$ In addition, hysteroscopy makes the observation of endometrial lesions 


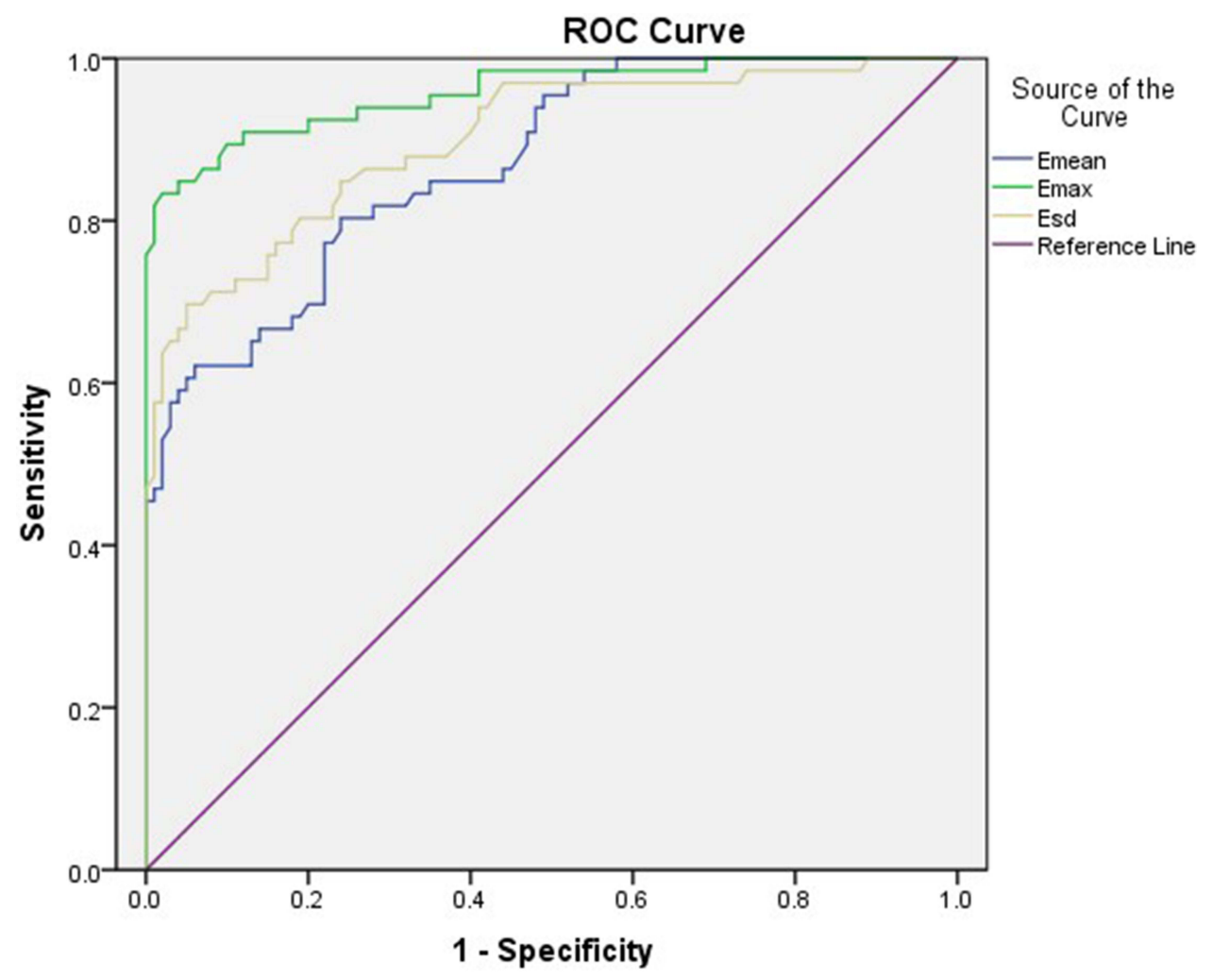

Diagonal segments are produced by ties.

Figure 2 Receiver operating characteristic curves of Emean, Emax and Esd to identify normal endometrial and endometrial cancer.

more intuitive, detailed and accurate. In recent years, endometrial biopsy under hysteroscopy has been increasingly widely used in the diagnosis of endometrial cancer, gradually replacing the traditional piecewise curettage. ${ }^{23,24}$ In the early 1990s, Ophir $^{25}$ and other scholars first proposed ultrasonic elasticity technology, which was considered as a new method for quantitative measurement of tissue hardness. Structures and components of various lesions are different, and result in varied tissue elasticity hardness. It is a new idea to judge the occurrence of diseases by measuring the elastic hardness of tissue. Realtime SWE can quantitatively obtain the elastic hardness of tissues, which makes this idea come true. In this study, real-time SWE was used to obtain the Emax, Emean and
Esd values of tissues or lesions. The Emax, Emean and Esd reflected the maximum tissular hardness, average hardness, and the variability of the tissular hardness of the whole measuring area.

Although the ages of the endometrial cancer group, the endometrial hyperplasia group, and the polyp group varied in this study (Table 3), the age at which the disease occurred was consistent. The patients in the normal endometrium group were all premenopausal women, and in order to ensure normal menstruation and no endometrial lesions, the selected patients in this group were younger.

In the SWE images, the proliferative endometrial group, secretory endometrial group, endometrial polyp group and endometrial hyperplasia group were

Table 5 Comparison of Diagnosis Value Among Different Methods of Emean, Emax, Esd

\begin{tabular}{|l|l|l|l|l|l|}
\hline & Sensitivity & Specificity & Positive Predictive Value & Negative Predictive Value & Accuracy \\
\hline Emean & $80.3 \%$ & $76.0 \%$ & $68.8 \%$ & $85.4 \%$ & $77.7 \%$ \\
Emax & $83.3 \%$ & $98.0 \%$ & $96.5 \%$ & $89.9 \%$ & $92.2 \%$ \\
Esd & $69.7 \%$ & $95.0 \%$ & $90.2 \%$ & $82.6 \%$ & $84.9 \%$ \\
$P$ & & & & & 0.01 \\
\hline
\end{tabular}


comparatively uniform blue, while the color of the endometrial cancer was mixed, as blue-yellow or even blueyellow-red. In quantitative diagnosis, there were no significant differences in the Emean and Emax values among the secretory endometrial group, endometrial polyp group and endometrial hyperplasia group. There was a significant difference between the proliferative endometrial group and the other three groups. The reason is that the stroma increased, and the glands decreased relatively in the proliferating stage, while the glands gradually increased and the stroma relatively decreased in the secretory stage. Endometrial polyps contain a large number of glands and thick-walled blood vessels. Endometrial hyperplasia is a proliferative change of glands and stroma. There was no significant difference in Esd values among the four groups, which may be related to the little difference in pathological composition of the four groups.

The Emean and Emax values of the endometrial carcinoma group were higher than those of the proliferative endometrial group, secretory endometrial group, endometrial polyp group and endometrial hyperplasia group. The differences had a statistical significance, in which endometrial cancer was more rigid than the other three groups. The reasons include: (1) the number of cancer cells is large. Previous studies on benign and malignant tumors in different organs have shown that the larger the number of tumor cells, the larger the ratio of nucleus to the cytoplasm and the greater the hardness of the tissue. ${ }^{17}$ (2) Fibrosis is obvious. Studies have shown that the more obvious the tissue fibrosis, the greater the tissue hardness. ${ }^{26}$ The fibrosis of endometrial cancer is obviously more than that of benign lesions and the normal endometrium, which leads to an increase in tissue hardness. (3) Neovascularization is abundant. Most of these vessels are arterioles, which can increase interstitial pressure and tissue hardness. (4) The growth mode is invasive growth, which is easy to adhere to the surrounding tissues, resulting in an increase in hardness. ${ }^{27-31}$ The Esd value of endometrial cancer is higher than that of the other four groups, which may be related to the different pathological composition of endometrial cancer and the other four groups.

It was found that the truncated values of Emean, Emax and Esd could differentiate and diagnose endometrial cancer. Emax has higher sensitivity, specificity and accuracy than other indicators, and the AUC of Emax is also higher than other indicators, indicating that Emax has the highest diagnostic value.
As a new generation of elastic imaging technology, real-time SWE technology can obtain quantitative indicators which makes up for the shortcomings of traditional elastic ultrasound. It can better display and identify the lesions and has good clinical value in the diagnosis and differential diagnosis of endometrial lesions.

However, this study has several limitations worth noting: (1) Because of the relatively small number of cases collected, there is no in-depth study on the differences between the different types of endometrial hyperplasia and endometrial cancer, which needs to be further explored under the large sample data in the future. (2) Because of the limited depth of real-time SWE measurements, unstable SWE maps and poor color are filled with endometrial lesions with a comparatively high uterine position or relatively large mass. The standard of data acquisition in this study is a depth of less than $4 \mathrm{~cm}$, which may lead to a deviation of data. (3) In the study, after multiple measurements, it was found that the thickness of the membrane was thinner after menopause and in the early proliferative period, and the SWE map was difficult to measure. Therefore, the thickness of the endometrium was $\geq 5 \mathrm{~mm}$. (4) This study uses transvaginal realtime shear wave elastography. Abdominal wall fat has little effect on vaginal ultrasound, so the impact of BMI on patients has not been studied, and it will be further explored in subsequent studies.

\section{Conclusion}

The application of Real-time SWE technology on the basis of traditional ultrasound can not only obtain quantitative indicators, but also provide new information of elastic hardness of tissue.

\section{Ethics Approval and Consent to Participate}

This study was conducted with approval from the Ethics Committee of the Fourth Hospital of Hebei Medical University. This study was conducted in accordance with the declaration of Helsinki. Written informed consent was obtained from all participants.

\section{Consent to Publish}

No sensitive participant information is included in the article. All participants signed written informed consents before inclusion in the study. 


\section{Acknowledgments}

We would like to acknowledge the hard and dedicated work of all the staff that implemented the intervention and evaluation components of the study.

\section{Funding}

This study was funded by the Funds for prevention and control of geriatric diseases in medical and health institutions directly under Hebei Provincial Department of Finance (JCYF2017-808). The funding body had no role in the design of the study and collection, analysis, and interpretation of data and in writing the manuscript.

\section{Disclosure}

The authors declare that they have no competing interests.

\section{References}

1. Soares CA, Pavan TZ, Miyaque AH, et al. Influence of pulse repetition frequency on 3-d power doppler quantification. Ultrasound Med Biol. 2016;42(12):2887-2892. doi:10.1016/j.ultrasmedbio.2016.08.015

2. Tacconi L, Giordan E, Signorelli F. Endoscopic approach technique for recurrent lumbar prolapsed disc. Neuro Quantol. 2019;17(5). doi:10.14704/nq.2019.17.5.2332

3. Filipchiuk $\mathrm{C}$, Laganà AS, Beteli R, et al. BIRC5/survivin expression as a non-invasive biomarker of endometriosis. Diagnostics (Basel). 2020;10(8):533. PMID: 32751449; PMCID: PMC7459871. doi:10.3390/diagnostics 10080533

4. Laganà AS, Vitale SG, Salmeri FM, et al. Unus pro omnibus, omnes pro uno: a novel, evidence-based, unifying theory for the pathogenesis of endometriosis. Med Hypotheses. 2017;103:10-20. PMID: 28571791. doi:10.1016/j.mehy.2017.03.032.

5. Yuan X, Saravelos SH, Wang Q, et al. Endometrial thickness as a predictor of pregnancy outcomes in 10787 fresh IVF-ICSI cycles. Reprod Biomed Online. 2016;33(2):197-205. doi:10.1016/j. rbmo.2016.05.002

6. Sebag F, Vaillant-Lombard J, Berbis J, et al. Shear wave elastography: a new ultrasound imaging mode for the differential diagnosis of benign and malignam thyroid nodules. $J$ Clin Endocrinol Metab. 2010;95:5281-5288. doi:10.1210/jc.2010-0766

7. Scioscia M, Virgilio BA, Laganà AS, et al. Differential diagnosis of endometriosis by ultrasound: a rising challenge. Diagnostics (Basel). 2020;10(10):848. PMID: 33092074; PMCID: PMC7589178. doi:10.3390/diagnostics 10100848

8. Noventa M, Scioscia M, Schincariol M, et al. Imaging modalities for diagnosis of deep pelvic endometriosis: comparison between trans-vaginal sonography, rectal endoscopy sonography and magnetic resonance imaging. A head-to-head meta-analysis. Diagnostics (Basel). 2019;9(4):225. PMID: 31861142; PMCID: PMC6963762. doi:10.3390/diagnostics9040225

9. Barra F, Biscaldi E, Scala C, et al. A Prospective Study comparing three-dimensional rectal water contrast transvaginal ultrasonography and computed tomographic colonography in the diagnosis of rectosigmoid endometriosis. Diagnostics (Basel). 2020;10(4):252. PMID: 32344709; PMCID: PMC7236009. doi:10.3390/diagnostics10040252

10. Scioscia M, Laganà AS, Caringella G, Guerriero S. Transvaginal strain elastosonography in the differential diagnosis of rectal endometriosis: some potentials and limits. Diagnostics (Basel). 2021;11 (1):99. PMID: 33435486; PMCID: PMC7826831. doi:10.3390/ diagnostics11010099
11. Tanter M, Bercoff J, Athanasiou A, et al. Quantitative assessment of breast lesion viscoelasticity: initial clinical results using supersonic shear imaging. Ultrasound Med Biol. 2008;34(9):1373-1386. doi:10.1016/j.ultrasmedbio.2008.02.002

12. Counade M, Pernot M, Prada C, et al. Quantitative assessment of arterial wall biomechanical properties using shear wave imaging. Ultrasound Med Biol. 2010;36(10):1662-1676. doi:10.1016/j. ultrasmedbio.2010.07.004

13. Garrard JW, Ramnarine K. Shear-wave elastography in carotid plaques; Comparison with grayscale median and histological assessment in an interesting case. Ultraschall Med. 2014;35(1):1-3. doi:10.1055/ s-0033-1350310

14. Ramnarine KV, Garrard JW, Kanber B, et al. Shear wave elastography imaging of carotid plaques: feasible, reproducible and of clinical potential. Cardiovasc Ultrasound. 2014;12(8):49. doi:10.1186/14767120-12-49

15. Suhr MC, Oledzka M. Considerations and intervention in congenital muscular torticollis. Curr Opin Pediatr. 2015;27(1):75-81. doi:10.1097/MOP.0000000000000175

16. Li X, Wang JN, Fan ZY, et al. Determination of the elasticity of breast tissue during the menstrual cycle using real-time shear wave elastography. Ultrasound Med Biol. 2015;41(12):3140-3147. doi:10.1016/j.ultrasmedbio.2015.07.013

17. Liu C, Li TT, Hu Z, et al. Transvaginal real-time shear wave elastography in the diagnosis of cervical disease. $J$ Ultrasound Med. 2019:1-9.

18. Tessarolo M, Bonino L, Camanni M, et al. Elastosonography: a possible new tool for diagnosis of adenomyosis? Eur Radiol. 2011;21:1546-1552. doi:10.1007/s00330-011-2064-z

19. Schiffmann ML, Schäfer SD, Schüring AN, et al. Importance of transvaginal ultrasound applying elastography for identifying deep infiltrating endometriosis - a feasibility study. Ultraschall Med. 2014;35:561-565. doi:10.1055/s-0034-1366747

20. Neale E, Housden R, Crawford R, et al. A pilot study using transvaginal real-time ultrasound elastography to evaluate the postmenopausal endometrium. Ultrasound Obstet Gynecol. 2011;38:233-236. doi:10.1002/uog.9057

21. Scioscia M, Noventa M, Laganà AS. Abnormal uterine bleeding and the risk of endometrial cancer: can subendometrial vascular ultrasound be of help to discriminate cancer from adenomyosis? Am J Obstet Gynecol. 2020;223:605-606. doi:10.1016/j.ajog.2020.05. 049

22. Laganà AS, Ciancimino L, Mancuso A, Chiofalo B, Rizzo $\mathrm{P}$, Triolo O. 3D sonohysterography vs hysteroscopy: a cross-sectional study for the evaluation of endouterine diseases. Arch Gynecol Obstet. 2014;290:1173-1178. doi:10.1007/s00404014-3366-6

23. Puente E, Alonso L, Laganà AS, Ghezzi F, Casarin J, Carugno J. Chronic endometritis: old problem, novel insights and future challenges. Int J Fertil Steril. 2019;13:250-256. doi:10.22074/ ijfs. 2020.5779

24. Chiofalo B, Mazzon I, Di Angelo Antonio S, et al. Hysteroscopic evaluation of endometrial changes in breast cancer women with or without hormone therapies: results from a Large Multicenter Cohort Study. J Minim Invasive Gynecol. 2020;27:832-839. doi:10.1016/j. jmig.2019.08.007

25. Ophir J, Cespedes I, Ponnekanti H, et al. Elastography: a quantitative method for imaging the elasticity of biological tissues. Ultrason Imaging. 1991;13(2):111-134. doi:10.1177/ 016173469101300201

26. Padoin AV, Mottin CC, Moretto M, et al. A comparison of wedge and needle hepatic biopsy in open bariatric surgery. Obes Surg. 2006;16:178-182. doi:10.1381/096089206775565159

27. Athanasiou A, Tardivon A, Tanter M, et al. Breast lesions: quantitative elastography with supersonic shear imaging-preliminary results. Radiology. 2010;256:297-303. doi:10.1148/radiol.10090385 
28. Hong Y, Liu X, Li Z, et al. Real-time ultrasound elastography in the differential diagnosis of benign and malignant thyroid nodules. $J$ Ultrasound Med. 2009;28:861-867. doi:10.7863/jum.2009. 28.7.861

29. Miyagawa T, Tsutsumi M, Matsumura T, et al. Real-time elastography for the diagnosis of prostate cancer: evaluation of elastographic moving images. Jpn J Clin Oncol. 2009;39:394-398. doi:10.1093/ jjco/hyp026
30. Kagoya R, Monobe H, Tojima H. Utility of elastography for differential diagnosis of benign and malignant thyroid nodules. Otolaryngol Head Neck Surg. 2010;143:230-234. doi:10.1016/j. otohns.2010.04.006

31. Regini E, Bagnera S, Tota D, et al. Role of sonoelastography in characterizing breast nodules. Preliminary experience with 120 lesions. Radiol Med. 2010;115(4):551-562. doi:10.1007/s11547010-0518-z

\section{Publish your work in this journal}

The International Journal of General Medicine is an international, peer-reviewed open-access journal that focuses on general and internal medicine, pathogenesis, epidemiology, diagnosis, monitoring and treatment protocols. The journal is characterized by the rapid reporting of reviews, original research and clinical studies across all disease areas. The manuscript management system is completely online and includes a very quick and fair peer-review system, which is all easy to use. Visit http://www.dovepress.com/ testimonials.php to read real quotes from published authors. 